\title{
Adaptive Robust Optimization for the Security Constrained Unit Commitment Problem
}

\author{
Dimitris Bertsimas, Member, IEEE, Eugene Litvinov, Senior Member, IEEE, Xu Andy Sun, Member, IEEE, \\ Jinye Zhao, Member, IEEE, and Tongxin Zheng, Senior Member, IEEE
}

\begin{abstract}
Unit commitment, one of the most critical tasks in electric power system operations, faces new challenges as the supply and demand uncertainty increases dramatically due to the integration of variable generation resources such as wind power and price responsive demand. To meet these challenges, we propose a two-stage adaptive robust unit commitment model for the security constrained unit commitment problem in the presence of nodal net injection uncertainty. Compared to the conventional stochastic programming approach, the proposed model is more practical in that it only requires a deterministic uncertainty set, rather than a hard-to-obtain probability distribution on the uncertain data. The unit commitment solutions of the proposed model are robust against all possible realizations of the modeled uncertainty. We develop a practical solution methodology based on a combination of Benders decomposition type algorithm and the outer approximation technique. We present an extensive numerical study on the real-world large scale power system operated by the ISO New England. Computational results demonstrate the economic and operational advantages of our model over the traditional reserve adjustment approach.
\end{abstract}

Index Terms-Bilevel mixed-integer optimization, power system control and reliability, robust and adaptive optimization, security constrained unit commitment.

\section{INTRODUCTION}

$\mathbf{U}$ NIT commitment (UC) is one of the most critical decision processes performed by system operators in deregulated electricity markets as well as in vertically integrated utilities. The objective of the UC problem is to find a unit commitment schedule that minimizes the commitment and dispatch costs of meeting the forecast system load, taking into account various physical, inter-temporal constraints for generating resources, transmission, and system reliability requirements.

During the normal real-time operation, system operator dispatches the committed generation resources to satisfy the actual demand and reliability requirements. In the event that the actual system condition significantly deviates from the expected condition, system operator needs to take corrective actions such as

Manuscript received March 16, 2011; revised September 18, 2011 and April 02, 2012; accepted May 25, 2012. Date of publication July 24, 2012; date of current version January 17, 2013. Paper no. TPWRS-00231-2011.

D. Bertsimas is with the Sloan School of Management and the Operations Research Center, Massachusetts Institute of Technology, Cambridge, MA 02139 USA (e-mail: dbertsim@mit.edu).

E. Litvinov, J. Zhao, and T. Zheng are with the Department of Business Architecture \& Technology, ISO New England, Holyoke, MA 01040 USA (e-mail: elitvinov@iso-ne.com; jzhao@iso-ne.com; tzheng@iso-ne.com).

X. A. Sun is with the H. Milton Stewart School of Industrial and Systems Engineering, Georgia Institute of Technology, Atlanta, GA 30332 USA (e-mail: sunx@mit.edu).

Digital Object Identifier 10.1109/TPWRS.2012.2205021 committing expensive fast-start generators, voltage reduction, or load shedding in emergency situation to maintain system security. The main causes of the unexpected events come from the uncertainties associated with the load forecast error, changes of system interchange schedules, generator's failure to follow dispatch signals, and unexpected transmission and generation outages.

In recent years, higher penetration of variable generation resources (such as wind power, solar power, and distributed generators) and more price-responsive demand participation have posed new challenges to the unit commitment process, especially in the independent system operator (ISO) managed electricity markets. It becomes important for the ISOs to have an effective methodology that produces robust unit commitment decisions and ensures the system reliability in the presence of increasing real-time uncertainty.

Previous studies of uncertainty management in the UC problem can be divided into two groups. The first group commits and dispatches generating resources to meet a deterministic forecast load, and handles uncertainty by imposing conservative reserve requirements. The second group relies on stochastic optimization techniques. The first group, the so-called reserve adjustment method, is widely used in today's power industry. Much of research along this vein, including [1]-[4], has focused on analyzing the levels of reserve requirements based on deterministic criteria, such as loss of the largest generator or system import change. Such an approach is easy to implement in practice. However, committing extra generation resources as reserves could be an economically inefficient way to handle uncertainty, especially when the reserve requirement is determined largely by some ad-hoc rules, rather than by a systematic analysis. Also, since the UC decision only considers the expected operating condition, even with enough reserve available, the power system may still suffer capacity inadequacy when the real-time condition, such as load, deviates significantly from the expected value. This is confirmed by the ISO's operational experience as well as by the numerical simulation shown later.

The stochastic optimization approach explicitly incorporates a probability distribution of the uncertainty, and often relies on pre-sampling discrete scenarios of the uncertainty realizations [5]-[9]. This approach has some practical limitations in the application to large scale power systems. First, it may be difficult to identify an accurate probability distribution of the uncertainty. Second, stochastic UC solutions only provide probabilistic guarantees to the system reliability. To obtain a reasonably high guarantee requires a large number of scenario samples, which results in a problem that is computationally inten- 
sive. To improve the robustness of stochastic UC solutions, Ruiz et al. [10] proposed a hybrid approach combining the reserve requirement and stochastic optimization methods. A recent work [11] proposed a framework that combines uncertainty quantification with stochastic optimization. This framework could also be integrated into the robust optimization formulation proposed below.

Robust optimization has recently gained substantial popularity as a modeling framework for optimization under parameter uncertainty, led by the work in [12]-[18]. The approach is attractive in several aspects. First, it only requires moderate information about the underlying uncertainty, such as the mean and the range of the uncertain data; and the framework is flexible enough that the modeler can always incorporate more probabilistic information such as correlation to the uncertainty model, when such information is available. For instance, the method of uncertainty quantification (UQ) proposed in [11] can be integrated into the robust optimization UC model, where the UQ module updates the uncertainty model as more information is obtained in time. Second, the robust model constructs an optimal solution that immunizes against all realizations of the uncertain data within a deterministic uncertainty set. This robustness is a desirable feature, especially when the penalty associated with infeasible solutions is very high, as the case in the power system operations. Hence, the concept of robust optimization is consistent with the risk-averse fashion in which the power systems are operated. Robust optimization has been broadly applied in engineering and management sciences, such as structural design, integrated circuit design, statistics, inventory management, to name a few. See [19] and references therein.

In this paper, we propose a two-stage adaptive robust optimization model for the security constrained unit commitment (SCUC) problem, where the first-stage UC decision and the second-stage dispatch decision are robust against all uncertain nodal net injection realizations. Furthermore, the second-stage dispatch solution has full adaptability to the uncertainty. The critical constraints such as network constraints, ramp rate constraints and transmission security constraints are incorporated into the proposed model as well. It is key to design a proper uncertainty set to control the conservatism of the robust solution. We use a special technique proposed in [17] and [18] for this purpose. We develop a practical solution method, and extensively test the method on a real-world power system. Papers [20] and [21] proposed similar robust optimization UC models. However, their proposals ignored reserve constraints and did not study critical issues such as the impact of robust solutions on system efficiency, operational stability, and robustness of the UC solutions. Our research was conducted independently of the work in [20] and [21]. The main contributions of our paper are summarized below.

1) We formulate a two-stage adaptive robust optimization model for the SCUC problem. Given a pre-specified nodal net injection uncertainty set, the two-stage adaptive robust UC model obtains an "immunized against uncertainty" first-stage commitment decision and a second-stage adaptive dispatch actions by minimizing the sum of the unit commitment cost and the dispatch cost under the worst-case realization of uncertain nodal net injection. The nodal net injection uncertainty set models variable resources such as non-dispatchable wind generation, real-time demand variation, and interchange uncertainty. The parameters in the uncertainty set provide control over the conservatism of the robust solution.

2) We develop a practical solution methodology to solve the adaptive robust model. In particular, we design a two-level decomposition approach. A Benders decomposition type algorithm is employed to decompose the overall problem into a master problem involving the first-stage commitment decisions at the outer level and a bilinear subproblem associated with the second-stage dispatch actions at the inner level, which is solved by an outer approximation approach [22], [23]. The proposed solution method applies to general polyhedral uncertainty sets. Computational study shows the efficiency of the method.

3) We conduct extensive numerical experiments on the realworld large scale power system operated by the ISO New England. We study the performance of the adaptive robust model and provide detailed comparison with the current practice, the reserve adjustment approach. Specifically, we analyze the merit of the adaptive robust model from three aspects: economic efficiency, contribution to real-time operation reliability, and robustness to probability distributions of the uncertainty.

The paper is organized as follows. Section II describes the deterministic SCUC formulation. Section III introduces the twostage adaptive robust SCUC formulation. Section IV discusses the solution methodology. Section V presents computational results, including a discussion on the proper way to choose the level of conservatism in the robust model. Section VI concludes with discussions.

\section{Deterministic SCUC PROBLEM}

The deterministic SCUC problem is extensively studied in the power system literature (e.g., [24], [25]). Please see the Appendix for a detailed model. Here we present a compact matrix formulation:

$$
\begin{array}{ll}
\min _{\boldsymbol{x}, \boldsymbol{y}} & \boldsymbol{c}^{T} \boldsymbol{x}+\boldsymbol{b}^{T} \boldsymbol{y} \\
\text { s.t. } & \boldsymbol{F} \boldsymbol{x} \leq \boldsymbol{f}, \\
& \boldsymbol{H} \boldsymbol{y} \leq \boldsymbol{h}, \\
& \boldsymbol{A} \boldsymbol{x}+\boldsymbol{B} \boldsymbol{y} \leq \boldsymbol{g}, \\
& \boldsymbol{I}_{u} \boldsymbol{y}=\overline{\boldsymbol{d}}, \\
& \boldsymbol{x} \text { binary. }
\end{array}
$$

The binary variable $\boldsymbol{x}$ is a vector of commitment related decisions including the on/off and start-up/shut-down status of each generation unit for each time interval of the commitment period, usually $24 \mathrm{~h}$ in an ISO setting. The continuous variable $y$ is a vector of dispatch related decision including the generation output, load consumption levels, resource reserve levels, and power flows in the transmission network for each time interval. By convention, generation, reserve, and flow take positive sign, whereas load takes negative sign. 
The objective function is to minimize the sum of commitment cost $\boldsymbol{c}^{T} \boldsymbol{x}$ (including start-up, no-load, and shut-down costs) and dispatch cost $\boldsymbol{b}^{T} \boldsymbol{y}$ over the planning horizon. Constraint (1), involving only commitment variables, contains minimum up and down, and start-up/shut-down constraints. Constraint (2) includes dispatch related constraints such as energy balance (equality can always be written as two opposite inequalities), reserve requirement, reserve capacity, transmission limit, and ramping constraints. Constraint (3) couples the commitment and dispatch decisions, including minimum and maximum generation capacity constraints. Constraint (4) emphasizes the fact that the uncertain nodal net injections are fixed at expected values in the deterministic model $\left(\boldsymbol{I}_{u}\right.$ selects the components from $\boldsymbol{y}$ that correspond to uncertain resources).

\section{Two-Stage Adaptive Robust SCUC Formulation}

In this section, we first discuss the uncertainty set, which is a key building block of the robust model. Then, we introduce the two-stage adaptive robust SCUC formulation and provide a detailed explanation.

The first step to build a robust model is to construct an uncertainty set. Unlike the stochastic optimization approach, the uncertainty model in a robust optimization formulation is not a probability distribution, but rather a deterministic set. In this paper, the uncertain parameter is the nodal net injection. We consider the following uncertainty set of nodal net injection at each time period $t$ in the planning horizon $\mathcal{T}$ :

$$
\begin{aligned}
\mathcal{D}^{t}\left(\overline{\boldsymbol{d}}^{t}, \hat{\boldsymbol{d}}^{t}, \Delta^{t}\right):=\left\{\boldsymbol{d}^{t} \in \mathbb{R}^{N_{d}}: \sum_{i \in \mathcal{N}_{d}} \frac{\left|d_{i}^{t}-\bar{d}_{i}^{t}\right|}{\hat{d}_{i}^{t}} \leq \Delta^{t}\right. \\
\left.d_{i}^{t} \in\left[\bar{d}_{i}^{t}-\hat{d}_{i}^{t}, \bar{d}_{i}^{t}+\hat{d}_{i}^{t}\right], \forall i \in \mathcal{N}_{d}\right\}
\end{aligned}
$$

where $\mathcal{N}_{d}$ is the set of nodes that have uncertain injections, $N_{d}$ is the number of such nodes, $\boldsymbol{d}^{t}=\left(d_{i}^{t}, i \in \mathcal{N}_{d}\right)$ is the vector of uncertain net injections at time $t, \bar{d}_{i}^{t}$ is the nominal value of the net injection of node $i$ at time $t, \hat{d}_{i}^{t}$ is the deviation from the nominal net injection value of node $i$ at time $t$, the interval $\left[\bar{d}_{i}^{t}-\hat{d}_{i}^{t}, \bar{d}_{i}^{t}+\hat{d}_{i}^{t}\right]$ is the range of the uncertain $d_{i}^{t}$, and the inequality in (5) controls the total deviation of all injections from their nominal values at time $t$. The parameter $\Delta^{t}$ is the "budget of uncertainty", taking values between 0 and $N_{d}$. When $\Delta^{t}=0$, the uncertainty set $\mathcal{D}^{t}=\left\{\overline{\boldsymbol{d}}^{t}\right\}$ is a singleton, corresponding to the nominal deterministic case. As $\Delta^{t}$ increases, the size of the uncertainty set $\mathcal{D}^{t}$ enlarges. This means that larger total deviation from the expected net injection is considered, so that the resulting robust $\mathrm{UC}$ solutions are more conservative and the system is protected against a higher degree of uncertainty. When $\Delta^{t}=N_{d}, \mathcal{D}^{t}$ equals to the entire hypercube defined by the intervals for each $d_{i}^{t}$ for $i \in \mathcal{N}_{d}$.

Now we formulate the two-stage adaptive robust SCUC model as follows:

$$
\begin{aligned}
\min _{\boldsymbol{x}, \boldsymbol{y}(\cdot)} & \left(\boldsymbol{c}^{T} \boldsymbol{x}+\max _{\boldsymbol{d} \in \mathcal{D}} \boldsymbol{b}^{T} \boldsymbol{y}(\boldsymbol{d})\right) \\
\text { s.t. } & \boldsymbol{F} \boldsymbol{x} \leq \boldsymbol{f}, \boldsymbol{x} \text { is binary } \\
& \boldsymbol{H} \boldsymbol{y}(\boldsymbol{d}) \leq \boldsymbol{h}(\boldsymbol{d}), \forall \boldsymbol{d} \in \mathcal{D} \\
& \boldsymbol{A x}+\boldsymbol{B} \boldsymbol{y}(\boldsymbol{d}) \leq \boldsymbol{g}, \forall \boldsymbol{d} \in \mathcal{D} \\
& \boldsymbol{I}_{u} \boldsymbol{y}(\boldsymbol{d})=\boldsymbol{d}, \forall \boldsymbol{d} \in \mathcal{D}
\end{aligned}
$$

where $\boldsymbol{d}=\left(\boldsymbol{d}^{t}, t \in \mathcal{T}\right)$ and $\mathcal{D}=\prod_{i \in \mathcal{T}} \mathcal{D}^{t}$. The objective function has two parts, reflecting the two-stage nature of the decision. The first part is the commitment cost. The second part is the worst case second-stage dispatch cost.

From this formulation, we can see that the commitment decision $\boldsymbol{x}$ takes into account all possible future net injection represented in the uncertainty set. Such a UC solution remains feasible, thus robust, for any realization of the uncertain net injection. In comparison, the traditional UC solution only guarantees feasibility for a single nominal net injection, whereas the stochastic optimization UC solution only considers a finite set of preselected scenarios of the uncertain net injection. Furthermore, in our formulation the optimal second-stage decision $\boldsymbol{y}(\boldsymbol{d})$ is a function of the uncertain net injection $\boldsymbol{d}$, therefore, fully adaptive to any realization of the uncertainty. Notice that $\boldsymbol{d}$ is also a function of the first-stage decision $\boldsymbol{x}$. However, we write it as $\boldsymbol{y}(\boldsymbol{d})$ to emphasize the adaptability of the second-stage decision to the uncertainty $\boldsymbol{d}$. The functional form of $\boldsymbol{y}(\cdot)$ is determined implicitly by the optimization problem, as opposed to being presumed as in the case of affinely adaptive policies (see [26] and discussion in the conclusion part of this paper). The full adaptability properly models the economic dispatch procedure in the real-time operation.

The above formulation can be recast in the following equivalent form, which is suitable for developing numerical algorithms:

$$
\begin{array}{ll}
\min _{\boldsymbol{x}} & \left(\boldsymbol{c}^{T} \boldsymbol{x}+\max _{\boldsymbol{d} \in \mathcal{D}} \min _{\boldsymbol{y} \in \Omega(\boldsymbol{x}, \boldsymbol{d})} \boldsymbol{b}^{T} \boldsymbol{y}\right) \\
\text { s.t. } & \boldsymbol{F} \boldsymbol{x} \leq \boldsymbol{f}, \quad \boldsymbol{x} \text { binary }
\end{array}
$$

where $\Omega(\boldsymbol{x}, \boldsymbol{d})=\left\{\boldsymbol{y}: \boldsymbol{H} \boldsymbol{y} \leq \boldsymbol{h}, \boldsymbol{A x}+\boldsymbol{B} \boldsymbol{y} \leq \boldsymbol{g}, \boldsymbol{I}_{u} \boldsymbol{y}=\boldsymbol{d}\right\}$ is the set of feasible dispatch solutions for a fixed commitment decision $\boldsymbol{x}$ and nodal net injection realization $\boldsymbol{d}$. Notice that the worst case dispatch cost has a max-min form, where $\min _{\boldsymbol{y} \in \Omega(\boldsymbol{x}, \boldsymbol{d})} \boldsymbol{b}^{T} \boldsymbol{y}$ determines the economic dispatch cost for a fixed commitment $\boldsymbol{x}$ and net injection $\boldsymbol{d}$, which is then maximized over the uncertainty set $\mathcal{D}$. A slack variable is included in the energy balance constraints in the inner max-min problem to ensure its feasibility.

It is useful to write out the dual of the dispatch problem $\min _{\boldsymbol{y} \in \Omega(\boldsymbol{x}, \boldsymbol{d})} \boldsymbol{b}^{T} \boldsymbol{y}$. Denote its cost by $S(\boldsymbol{x}, \boldsymbol{d})$ :

$$
\begin{aligned}
S(\boldsymbol{x}, \boldsymbol{d})= & \max _{\boldsymbol{\varphi}, \boldsymbol{\lambda}, \boldsymbol{\eta}} \boldsymbol{\lambda}^{T}(\boldsymbol{A x}-\boldsymbol{g})-\boldsymbol{\varphi}^{T} \boldsymbol{h}+\boldsymbol{\eta}^{T} \boldsymbol{d} \\
& -\boldsymbol{\lambda}^{T} \boldsymbol{B}-\boldsymbol{\varphi}^{T} \boldsymbol{H}+\boldsymbol{\eta}^{T} \boldsymbol{I}_{u}=\boldsymbol{b}^{T} \\
& \boldsymbol{\varphi} \geq \mathbf{0}, \boldsymbol{\lambda} \geq \mathbf{0}, \boldsymbol{\eta} \text { free }
\end{aligned}
$$

where $\varphi, \lambda$, and $\boldsymbol{\eta}$ are the multipliers of the constraints (2)-(4), respectively.

Now, the second-stage problem $\max _{\boldsymbol{d} \in \mathcal{D}} \min _{\boldsymbol{y} \in \Omega(\boldsymbol{x}, \boldsymbol{d})} \boldsymbol{b}^{T} \boldsymbol{y}$ is equivalent to a bilinear optimization problem given as follows:

$$
\begin{gathered}
R(\boldsymbol{x})=\max _{\boldsymbol{d}, \boldsymbol{\varphi}, \boldsymbol{\lambda}, \boldsymbol{\eta}} \boldsymbol{\lambda}^{T}(\boldsymbol{A x}-\boldsymbol{g})-\boldsymbol{\varphi}^{T} \boldsymbol{h}+\boldsymbol{\eta}^{T} \boldsymbol{d} \\
-\boldsymbol{\lambda}^{T} \boldsymbol{B}-\boldsymbol{\varphi}^{T} \boldsymbol{H}+\boldsymbol{\eta}^{T} \boldsymbol{I}_{u}=\boldsymbol{b}^{T} \\
\boldsymbol{\varphi} \geq \mathbf{0}, \boldsymbol{\lambda} \geq \mathbf{0}, \boldsymbol{\eta} \text { free, } \boldsymbol{d} \in \mathcal{D}
\end{gathered}
$$

where the constraints involving variable $z:=(\boldsymbol{\varphi}, \boldsymbol{\lambda}, \boldsymbol{\eta})$ define a polyhedral set, denoted as $\mathcal{E}$. Due to the bilinear structure of the objective function, the optimal solution of problem 
$(9) z^{*}:=\left(\boldsymbol{\varphi}^{*}, \boldsymbol{\lambda}^{*}, \boldsymbol{\eta}^{*}\right)$ is an extreme point of the polyhedron $\mathcal{E}$, and similarly the optimal solution $\boldsymbol{d}^{*}$ is an extreme point of $\mathcal{D}$. Therefore, if we denote all the extreme points of $\mathcal{E}$ as $z_{1}, \ldots, z_{m}$, and all the extreme points of $\mathcal{D}$ as $\boldsymbol{d}_{1}, \ldots, \boldsymbol{d}_{k}, R(\boldsymbol{x})$ can be written equivalently as

$$
R(\boldsymbol{x})=\max _{\boldsymbol{z}_{i}, \forall i, \boldsymbol{d}_{j}, \forall j} \boldsymbol{\lambda}_{i}^{T}(\boldsymbol{A} \boldsymbol{x}-\boldsymbol{g})-\boldsymbol{\varphi}_{i}^{T} \boldsymbol{h}+\boldsymbol{\eta}_{i}^{T} \boldsymbol{d}_{j}
$$

which shows that $R(\boldsymbol{x})$ is the maximum of a finitely many affine functions in $\boldsymbol{x}$, hence $R(\boldsymbol{x})$ is a convex piecewise linear function in $\boldsymbol{x}$. However, in general we have no knowledge of the extreme points of $\mathcal{E}$ and $\mathcal{D}$, and computing $R(\boldsymbol{x})$ is non-trivial. To see this, notice that the objective function of (9) contains a non-concave bilinear term $\boldsymbol{\eta}^{T} \boldsymbol{d}$, and bilinear programs are in general NP-hard to solve. Another way to see this is from the formulation (7), where $R(\boldsymbol{x})$ can be written equivalently as

$$
R(\boldsymbol{x})=\max _{\boldsymbol{d} \in \mathcal{D}} \min _{\boldsymbol{y} \in \Omega(\boldsymbol{x}, \boldsymbol{d})} \boldsymbol{b}^{T} \boldsymbol{y} .
$$

Notice that the objective value of the inner problem $\min _{\boldsymbol{y} \in \Omega(\boldsymbol{x}, \boldsymbol{d})} \boldsymbol{b}^{T} \boldsymbol{y}$ is a convex function in $\boldsymbol{d}$. Therefore, evaluating $R(\boldsymbol{x})$ needs to maximize a convex function, which is generally NP-hard. Throughout the paper, we assume $R(\boldsymbol{x})<+\infty$ for all feasible $\boldsymbol{x}$. This can be ensured by adding penalty terms in the dispatch constraints. We omit the penalty terms here for a clear presentation.

\section{Solution Method to Solve THE ADAPTIVE ROBUST MODEL}

As analyzed in the previous section, the adaptive robust formulation (7) is a two-stage problem. The first-stage is to find an optimal commitment decision $\boldsymbol{x}$. The second-stage is to find the worst-case dispatch cost under a fixed commitment solution. Naturally, we will have a two-level algorithm. The outer level employs a Benders decomposition (BD) type cutting plane algorithm to obtain $\boldsymbol{x}$ using the information (i.e., cuts) generated from the inner level, which approximately solves the bilinear optimization problem (9).

\section{A. Outer Level: Benders Decomposition Algorithm}

The Benders decomposition algorithm is described below. Initialization: Let $\boldsymbol{x}_{0}$ be a feasible first-stage solution. Solve $R\left(\boldsymbol{x}_{0}\right)$ defined by (9) to get an initial solution $\left(\boldsymbol{d}_{1}, \boldsymbol{\varphi}_{1}, \boldsymbol{\lambda}_{1}, \boldsymbol{\eta}_{1}\right)$. Set the outer level lower bound $L^{B D}=-\infty$, upper bound $U^{B D}=+\infty$ and the number of iteration $k=1$. Choose an outer level convergence tolerance level $\epsilon(>0)$.

Iteration $k \geq 1$ :

Step 1) Solve BD master problem.

The master problem of BD is the following mixed integer program (MIP):

$$
\begin{array}{ll}
\min _{\boldsymbol{x}, \alpha} & \boldsymbol{c}^{T} \boldsymbol{x}+\alpha \\
\text { s.t. } & \alpha \geq \boldsymbol{\lambda}_{l}^{T}(\boldsymbol{A} \boldsymbol{x}-\boldsymbol{g})-\boldsymbol{\varphi}_{l}^{T} \boldsymbol{h}+\boldsymbol{\eta}_{l}^{T} \boldsymbol{d}_{l}, \forall l \leq k \\
& \boldsymbol{F} \boldsymbol{x} \leq \boldsymbol{f}, \boldsymbol{x} \text { binary. }
\end{array}
$$

Let $\left(\boldsymbol{x}_{k}, \alpha_{k}\right)$ be the optimum. Set $L^{B D}=\boldsymbol{c}^{T} \boldsymbol{x}_{k}+$ $\alpha_{k}$.

Step 2) Solve BD subproblem $R\left(\boldsymbol{x}_{k}\right)$.

We will discuss the methodology to solve $R\left(\boldsymbol{x}_{k}\right)$ in the next subsection. Let $\left(\boldsymbol{d}_{k+1}, \boldsymbol{\varphi}_{k+1}, \boldsymbol{\lambda}_{k+1}, \boldsymbol{\eta}_{k+1}\right)$ be the optimal solution. Set $U^{B D}=\boldsymbol{c}^{T} \boldsymbol{x}_{k}+R\left(\boldsymbol{x}_{k}\right)$.

Step 3) Check the outer level convergence.

If $U^{B D}-L^{B D}<\epsilon$, stop and return $\boldsymbol{x}_{k}$. Otherwise, let $k=k+1$, and go to step 1 .

To speed up the convergence of the above BD algorithm, we find it helpful to add dispatch constraints $\Omega\left(\boldsymbol{x}_{k}, \boldsymbol{d}_{k}\right)$ to the BD master problem (11) at certain iteration $k$ when $U^{B D}$ or $L^{B D}$ has improved slowly.

\section{B. Inner Level: Solve $R(\boldsymbol{x})$}

An outer approximation (OA) algorithm [22], [23] is used to solve the bilinear program (9), where the bilinear term in the objective is linearized around intermediate solution points and linear approximations are added to the OA formulation. Since the problem (9) is nonconcave, only a local optimum is guaranteed by the OA algorithm. To verify the quality of the solution, we test on different initial conditions and observe fast convergence and consistent results. The OA algorithm is described below.

Initialization: Fix the unit commitment decision $\boldsymbol{x}_{k}$ passed from the $k$ th iteration of the outer level BD algorithm. Find an initial net injection $\boldsymbol{d}_{1} \in \mathcal{D}$. Set the inner level lower bound $L^{O A}=-\infty$, upper bound $U^{O A}=+\infty$ and number of iteration $j=1$. Choose an inner level convergence tolerance level $\delta(>0)$.

Iteration $j \geq 1$ :

Step 1) Solve OA subproblem $S\left(\boldsymbol{x}_{k}, \boldsymbol{d}_{j}\right)$.

Solve $S\left(\boldsymbol{x}_{k}, \boldsymbol{d}_{j}\right)$, the dual dispatch problem defined by (8). Let $\left(\boldsymbol{\varphi}_{j}, \boldsymbol{\lambda}_{j}, \boldsymbol{\eta}_{j}\right)$ be the optimal solution. Set $L^{O A}=S\left(\boldsymbol{x}_{k}, \boldsymbol{d}_{j}\right)$. Define $L_{j}(\boldsymbol{d}, \boldsymbol{\eta})$, the linearization of the bilinear term $\boldsymbol{\eta}^{T} \boldsymbol{d}$ at $\left(\boldsymbol{d}_{j}, \boldsymbol{\eta}_{j}\right)$, as follows:

$$
L_{j}(\boldsymbol{d}, \boldsymbol{\eta})=\boldsymbol{\eta}_{j}^{T} \boldsymbol{d}_{j}+\left(\boldsymbol{\eta}-\boldsymbol{\eta}_{j}\right)^{T} \boldsymbol{d}_{j}+\left(\boldsymbol{d}-\boldsymbol{d}_{j}\right)^{T} \boldsymbol{\eta}_{j}
$$

Step 2) Check the inner level convergence. If $U^{O A}-$ $L^{O A}<\delta$, then stop and output the current solution. Otherwise, set $j=j+1$, go to Step 3 of the OA algorithm.

Step 3) Solve OA master problem.

Solve the linearized version of $R\left(\boldsymbol{x}_{k}\right)$, defined in the following:

$$
\begin{aligned}
U\left(\boldsymbol{d}_{j}, \boldsymbol{\eta}_{j}\right)= & \max _{\boldsymbol{d}, \boldsymbol{\varphi}, \boldsymbol{\lambda}, \boldsymbol{\eta}, \beta} \boldsymbol{\lambda}^{T}(\boldsymbol{A x}-\boldsymbol{g})-\boldsymbol{\varphi}^{T} \boldsymbol{h}+\beta \\
& \beta \leq L_{i}(\boldsymbol{d}, \boldsymbol{\eta}), \quad \forall i=1, \ldots, j \\
& -\boldsymbol{\lambda}^{T} \boldsymbol{B}-\boldsymbol{\varphi}^{T} \boldsymbol{H}+\boldsymbol{\eta}^{T} \boldsymbol{I}_{u}=\boldsymbol{b}^{T}, \boldsymbol{d} \in \mathcal{D} \\
& \boldsymbol{\varphi} \geq \mathbf{0}, \boldsymbol{\lambda} \geq \mathbf{0}, \boldsymbol{\eta} \text { free. }
\end{aligned}
$$

Since the uncertainty set $\mathcal{D}$ is assumed to be polyhedral, $U\left(\boldsymbol{d}_{j}, \boldsymbol{\eta}_{j}\right)$ is a linear program. Denote $\left(\boldsymbol{d}_{j+1}, \boldsymbol{\varphi}_{j+1}, \boldsymbol{\lambda}_{j+1}, \boldsymbol{\eta}_{j+1}, \beta_{j+1}\right)$ as the op- 


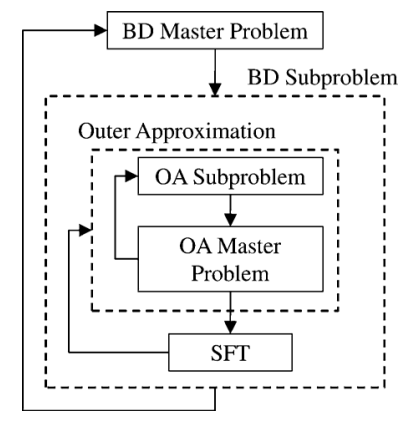

Fig. 1. Flow chart of the proposed two-level algorithm. SFT stands for simultaneous feasibility test, where security constraints, such as $N-1$ constraints, are tested at current solutions and violated constraints are added sequentially.

timal solution. Set the inner level upper bound as $U^{O A}=U\left(\boldsymbol{d}_{j}, \boldsymbol{\eta}_{j}\right)$.

We want to remark that the above inner level algorithm works for general polyhedral uncertainty set $\mathcal{D}$, not restricted to the budgeted uncertainty set of (5). The next theorem shows that the Benders cuts generated by the inner level are valid cuts.

Theorem 1: The dual solutions, $\left(\boldsymbol{d}_{j}, \boldsymbol{\varphi}_{j}, \boldsymbol{\lambda}_{j}, \boldsymbol{\eta}_{j}, \beta_{j}\right)$, of the $j$ th inner level problem generate valid inequalities for the secondstage value function $R(\boldsymbol{x})$, i.e.,

$$
R(\boldsymbol{x}) \geq \boldsymbol{\lambda}_{j}^{T}(\boldsymbol{A x}-\boldsymbol{g})-\boldsymbol{\varphi}_{j}^{T} \boldsymbol{h}+\boldsymbol{\eta}_{j}^{T} \boldsymbol{d}_{j}, \forall \boldsymbol{x} .
$$

Proof: The dual solutions $\boldsymbol{z}_{j}=\left(\boldsymbol{\varphi}_{j}, \boldsymbol{\lambda}_{j}, \boldsymbol{\eta}_{j}\right)$ generated by the OA subproblem $S\left(\boldsymbol{x}_{k}, \boldsymbol{d}_{j}\right)$ are extreme points of the dual polytope $\mathcal{E}$, because $S\left(\boldsymbol{x}_{k}, \boldsymbol{d}_{j}\right)$ is a linear optimization problem over $\mathcal{E}$. Therefore, using (10), we have

$$
\begin{aligned}
R(\boldsymbol{x}) & \geq \max _{\boldsymbol{d} \in \mathcal{D}} \boldsymbol{\lambda}_{j}^{T}(\boldsymbol{A x}-\boldsymbol{g})-\boldsymbol{\varphi}_{j}^{T} \boldsymbol{h}+\boldsymbol{\eta}_{j}^{T} \boldsymbol{d} \\
& \geq \boldsymbol{\lambda}_{j}^{T}(\boldsymbol{A x}-\boldsymbol{g})-\boldsymbol{\varphi}_{j}^{T} \boldsymbol{h}+\boldsymbol{\eta}_{j}^{T} \boldsymbol{d}_{j}
\end{aligned}
$$

which shows that the Benders cuts added to the BD master problem are valid cuts, i.e., the epigraph of $R(x)$ is above the Benders cuts.

The overall algorithmic framework is summarized in Fig. 1. In our implementation, we include all contingency transmission constraints in the BD subproblem.

\section{COMPUTATIONAL EXPERIMENTS}

In this section, we present a computational study to evaluate the performance of the adaptive robust (AdaptRob) approach and the reserve adjustment (ResAdj) approach. We test on the power system operated by the ISO New England Inc. (ISO-NE). We have the following data and uncertainty model.

The system and market data: The system has 312 generating units, 174 loads, and 2816 nodes. We select 4 representative transmission constraints that interconnect four major load zones in the ISO-NE's system (we also demonstrate the performance of our algorithms with 1876 transmission constraints). The market data is taken from a normal winter day of the ISO-NE's day-ahead energy market. In particular, we have 24-h data of generators' offer curves, reserve offers, expected nodal load, system reserve requirement (10-min spinning reserve, 10-min non-spinning reserve, and 30-min reserve), and various network parameters. The average total generation capacity per hour is $31929 \mathrm{MW}$ and the average forecast load per hour is $16232 \mathrm{MW}$.

The uncertainty model: We use the budgeted uncertainty set defined in (5), in which $\bar{d}_{j}^{t}$ is the nominal load given in the data. We set the range of load variation to be $\hat{d}_{j}^{t}=0.1 \bar{d}_{j}^{t}$ for each load $j$ at time $t$. The budget of uncertainty $\Delta^{t}$ takes values in the entire range of 0 to $N_{d}$. We will discuss the proper way to choose $\Delta^{t}$ within this range that results in the best performance of the robust solution in the following subsection.

For the ResAdj approach, we solve the deterministic UC problems presented in Section II at the expected load level with adjusted reserve requirement. In particular, we model the reserve adjustment as follows:

$$
q_{j, t}=q_{j, t}^{0}+\frac{\Delta^{t}}{N_{d}} \sum_{i=1}^{N_{d}} \hat{d}_{i}^{t}
$$

where $q_{j, t}$ is the system reserve requirement of type $j$ at time $t$, composed of the basic reserve level $q_{j, t}^{0}$ and an adjustment part proportional to the total variation of load. Here, the uncertainty budget $\Delta^{t}$ also controls the level of conservatism of the ResAdj solution.

The computational experiments proceed as follows.

1) Obtain UC solutions: Solve the ResAdj and AdaptRob UC models, respectively, for different uncertainty budgets: $\Delta^{t} \in\left[0, N_{d}\right]$.

2) Dispatch simulation: For each UC solution, solve the dispatch problem repeatedly for two sets of 1000 randomly generated loads.

One set of randomly sampled load follows a uniform distribution in the interval $\left[\bar{d}_{j}^{t}-\hat{d}_{j}^{t}, \bar{d}_{j}^{t}+\hat{d}_{j}^{t}\right]$ for each load $j$ at time $t$. The other set follows a normal distribution with mean $\bar{d}_{j}^{t}$ and standard deviation $\hat{d}_{j}^{t} / 1.44$, which results in an 85 percentile of load falling between $\left[\bar{d}_{j}^{t}-\hat{d}_{j}^{t}, \bar{d}_{j}^{t}+\hat{d}_{j}^{t}\right]$ and the load is truncated for nonnegative values. Notice that samples from both sets may fall outside of the budgeted uncertainty set, especially when the budget $\Delta^{t}$ is close to 0 . In this way, we can test the performance of the robust formulation when the uncertainty model is inaccurate.

To mimic the high cost of dispatching fast-start units or load shedding in the real-time operation, we introduce a slack variable $\boldsymbol{\gamma}$ to energy balance, reserve requirement, and transmission constraints in the dispatch simulation. If the real-time dispatch incurs any energy deficiency, reserve shortage, or transmission violation, at least one component of $\boldsymbol{\gamma}$ will be positive. The dispatch cost is the sum of production cost and penalty cost, i.e., $\boldsymbol{b}^{T} \boldsymbol{y}+\boldsymbol{\kappa}^{T} \boldsymbol{\gamma}$, where $\boldsymbol{\kappa}$ is set to $\$ 5000 / \mathrm{MWh}$ for each component.

The proposed two-level algorithm for the two-stage adaptive robust model is implemented in GAMS. The mixed integer program and linear program in the algorithm are solved with CPLEX 12.1.0 on a PC laptop with an Intel Core(TM) 2Duo 2.50-GHz CPU and $3 \mathrm{~GB}$ of memory. We set the convergence tolerance for the outer level BD algorithm to be $\epsilon=10^{-4}$, and the convergence tolerance for the inner level algorithm to be $\delta=10^{-3}$. The MIP gap for the BD master problem is set to $10^{-5}$. The average computation time to solve the robust UC problem is $6.14 \mathrm{~h}$. The average computation time for the reserve adjustment approach is $1.65 \mathrm{~h}$. The computational results 
presented in the following subsections use the above tolerance levels and MIP gap. If we relax the BD convergence tolerance $\epsilon$ to $10^{-3}$ and set the MIP gap of the BD master problem to be $10^{-3}$, the average computation time to solve the robust UC problem significantly decreases to $1.46 \mathrm{~h}$ with an average of $0.17 \%$ increase in terms of the worst-case total cost.

We compare the performance of the adaptive robust approach and the reserve adjustment approach in three aspects: 1) The average dispatch and total costs, 2) the volatility of these costs, and 3 ) the sensitivity of the these costs to different probability distributions of the uncertain load. The average cost indicates the economic efficiency of the UC decision; the volatility of these costs indicates the reliability of the real-time dispatch operation under the UC decision; the third aspect indicates how robust the UC decision is against load probability distributions. The performances of both approaches with respect to these three aspects are also compared under different levels of uncertain load variation.

The main conclusion is that a) by properly adjusting the budget level of the uncertainty set, the adaptive robust solution has lower average dispatch and total costs, indicating better economic efficiency of the robust approach; b) the adaptive robust solution significantly reduces the volatility of the total costs, as well as the penalty cost in the dispatch operation; c) the adaptive robust solution is significantly more robust to different probability distributions of load; d) the advantages of the adaptive robust solution are amplified when the level of load variation is higher.

We want to remark that point a) is quite contrary to the general impression that robust optimization is always conservative. In fact, by choosing a proper uncertainty level using probability laws, the robust solution achieves better economic efficiency than the conventional approach. We will present detailed discussion below. The computational results for normally and uniformly distributed loads are similar in illustrating a) and b). To be compact, we only present the results for normally distributed loads. For c), we compare the results of the two distributions. For d), we increase the level of load variation, and discuss the corresponding computational results in three performance aspects of cost efficiency, operational reliability and robustness to the underlying distribution.

\section{A. Cost Efficiency and the Choice of the Budget Level}

Table I reports the average dispatch costs and total costs of AdptRob and ResAdj solutions for normally distributed load when the uncertainty budget $\Delta^{t}$ varies from 0 to $N_{d}$. We can see that the AdptRob has lower average dispatch costs for all values of $\Delta^{t}$. The average total costs of the two approaches are more comparable.

To quantify the comparison, we define thecost saving as (ResAdj cost - AdptRob cost)/(ResAdj cost). For the normally distributed load, the AdptRob approach always has lower average dispatch cost than the ResAdj approach, and can save up to $2.7 \%$ or $\$ 472 \mathrm{k}$ (at $\Delta^{t}=0.1 N_{d}$ ), which is a significant saving for a daily operation. The total cost saving of the AdptRob approach ranges from $-0.84 \%$ (at $\Delta^{t}=0.4 N_{d}$ ) to $1.19 \%$ (at $\Delta^{t}=0.1 N_{d}$ ). Since the AdptRob approach protects the system against the worst-case load realization, in
TABLE I

Average Dispatch Costs And Total Costs of the ADPTROB AND RESADJ FOR NORMALLY DisTRIBUTED LOAD FOR $\Delta^{t} / N_{d}=0,0.1, \ldots, 1$ AND $\hat{d}_{j}^{t}=0.1 \bar{d}_{j}^{t}$

\begin{tabular}{c|cc|cc}
\hline & \multicolumn{2}{|c|}{ AdptRob } & \multicolumn{2}{c}{ ResAdj } \\
\hline budget & dispatch cost & total cost & dispatch cost & total cost \\
$\Delta^{t} / N_{d}$ & $(\mathrm{M} \$)$ & $(\mathrm{M} \$)$ & $(\mathrm{M} \$)$ & $(\mathrm{M} \$)$ \\
\hline 0.0 & 19.3530 & 20.8503 & 19.3530 & 20.8503 \\
0.1 & 16.9852 & 18.7290 & 17.4581 & 18.9547 \\
0.2 & 17.0513 & 18.8265 & 17.2391 & 18.7400 \\
0.3 & 17.0949 & 18.8773 & 17.2563 & 18.7595 \\
0.4 & 17.1448 & 18.9425 & 17.2570 & 18.7843 \\
0.5 & 17.1583 & 18.9569 & 17.2893 & 18.8250 \\
0.6 & 17.1705 & 18.9723 & 17.3030 & 18.8506 \\
0.7 & 17.1719 & 18.9896 & 17.3537 & 18.9062 \\
0.8 & 17.1715 & 18.9892 & 17.3899 & 18.9472 \\
0.9 & 17.1655 & 18.9898 & 17.3990 & 18.9669 \\
1.0 & 17.1652 & 18.9894 & 17.4524 & 19.0660 \\
\hline
\end{tabular}

TABLE II

Average Dispatch Costs and Total Costs of the ADPTROB AND RESADJ FOR NORMALLY DISTRIBUTED LOAD $\Delta^{t} / \sqrt{N_{d}}=0.5,1, \ldots, 3$ AND $\hat{d}_{j}^{t}=0.1 \bar{d}_{j}^{t}$

\begin{tabular}{c|cc|cc}
\hline & \multicolumn{2}{|c|}{ AdptRob } & \multicolumn{2}{c}{ ResAdj } \\
\hline $\begin{array}{c}\text { budget } \\
\Delta^{t} / \sqrt{N_{d}}\end{array}$ & $\begin{array}{c}\text { dispatch cost } \\
(\mathrm{M} \$)\end{array}$ & $\begin{array}{c}\text { total cost } \\
(\mathrm{M} \$)\end{array}$ & $\begin{array}{c}\text { dispatch cost } \\
(\mathrm{M} \$)\end{array}$ & $\begin{array}{c}\text { total cost } \\
(\mathrm{M} \$)\end{array}$ \\
\hline 0.5 & 16.9195 & 18.6050 & 18.1855 & 19.6837 \\
1.0 & 16.9650 & 18.6688 & 17.4907 & 18.9942 \\
1.5 & 16.9815 & 18.7365 & 17.3027 & 18.8006 \\
2.0 & 17.0297 & 18.7937 & 17.7403 & 19.2415 \\
2.5 & 17.0586 & 18.8366 & 17.6567 & 19.1618 \\
3.0 & 17.0745 & 18.8526 & 18.0804 & 19.5889 \\
\hline
\end{tabular}

general it commits more generating resources than the reserve adjustment approach, which considers uncertainty by adjusting the system reserve levels and does not identify the specific worst-case scenario.

For both average dispatch and total costs, we observe that the robust solution performs best when the budget level $\Delta^{t}$ is relatively small, e.g., around $0.1 N_{d}$. This phenomenon can actually be explained by the probability law, namely the central limit theorem. When a large number $N_{d}$ of random loads, independent as we assume, are aggregated, the volatility of the total load scales according to $\sqrt{N_{d}}$. Therefore, a proper level of uncertainty budget in the uncertainty set (5) should be chosen as $\Delta^{t} \sim$ $O\left(\sqrt{N_{d}}\right)$. Table II shows the results for $\Delta^{t}$ in $0.5 \sqrt{N_{d}}$ to $3 \sqrt{N_{d}}$, equivalently $\Delta^{t}$ equals $0.038 N_{d}$ to $0.227 N_{d}$, for $N_{d}=174$ in our system. We can see that, in this range of the uncertainty budget, the AdptRob has an even higher saving: the average dispatch cost saving from $1.86 \%$ or $321.2 \mathrm{k}$ (at $\Delta^{t}=1.5 \sqrt{N_{d}}$ ) to $6.96 \%$ or 1.27 Million (at $\Delta^{t}=0.5 \sqrt{N_{d}}$ ), and the average total cost saving from $0.34 \%$ or $64.1 \mathrm{k}$ (at $\Delta^{t}=1.5 \sqrt{N_{d}}$ ) to $5.48 \%$ or 1.08 Million (at $\Delta^{t}=0.5 \sqrt{N_{d}}$ ). This demonstrates that we can choose a proper uncertainty level by using the probability law as a guideline as opposed to always hedging against the most extreme scenarios. The robust UC solution with a proper uncertainty level obtains favorable economic performance and reduces the overall conservativeness of the robust model.

\section{B. Reliability of Dispatch Operation}

The adaptive robust approach also greatly reduces the volatility of the real-time dispatch costs. Table III shows the standard deviation (std) of the dispatch costs of the two approaches, and their ratios (ResAdj/AdptRob) for normally 
TABLE III

Standard DeViation of the Dispatch Costs of the Two ApProaches AND THEIR RATIO FOR NORMALLY DisTRIBUTED LOAD

\begin{tabular}{c|ccc}
\hline & AdptRob & ResAdj & ResAdj \\
\hline budget & $\begin{array}{c}\text { std dispatch } \\
\text { cost }(\mathbf{k} \$)\end{array}$ & $\begin{array}{c}\text { std dispatch } \\
\text { cost }(\mathbf{k} \$)\end{array}$ & $\begin{array}{c}\text { / } \\
\text { AdptRob }\end{array}$ \\
$\Delta^{t} / N_{d}$ & $1,769.5107$ & $\mathbf{1 , 7 6 9 . 5 1 0 7}$ & 1 \\
\hline 0.0 & 47.4900 & 687.5098 & $\mathbf{1 4 . 4 8}$ \\
0.1 & 46.3647 & 687.5098 & 8.62 \\
0.2 & 45.4248 & 377.7901 & 8.32 \\
0.3 & 44.2397 & 366.7359 & 8.29 \\
0.4 & 44.1075 & 377.1875 & 8.55 \\
0.5 & 43.9936 & 370.8673 & 8.43 \\
0.6 & 43.9263 & 377.0631 & 8.58 \\
0.7 & 43.9338 & 370.7203 & 8.44 \\
0.8 & 43.9023 & 357.9338 & 8.15 \\
0.9 & 43.9431 & 361.0376 & 8.22 \\
1.0 & & & \\
\hline
\end{tabular}

TABLE IV

Penalty Costs of AdPtRob and ResAdj ApProaches FOR NORMALLY DISTRIBUTED LOAD

\begin{tabular}{cc|cc}
\hline & AdptRob & \multicolumn{2}{|c}{ ResAdj } \\
\hline budget & $\begin{array}{c}\text { penalty } \\
\text { cost }(\mathrm{k} \$)\end{array}$ & $\begin{array}{c}\text { penalty } \\
\text { cost }(\mathrm{k} \$)\end{array}$ & $\begin{array}{c}\text { percent of } \\
\text { disp. cost }\end{array}$ \\
$\Delta^{t} / N_{d}$ & 2377.62 & $2,377.6237$ & $12.29 \%$ \\
\hline 0.0 & 0 & 497.8243 & $2.85 \%$ \\
0.1 & 0 & 272.4362 & $1.58 \%$ \\
0.2 & 0 & 268.0298 & $1.55 \%$ \\
0.3 & 0 & 252.3463 & $1.46 \%$ \\
0.4 & 0 & 267.6559 & $1.55 \%$ \\
0.5 & 0 & 259.7954 & $1.50 \%$ \\
0.6 & 0 & 267.6559 & $1.54 \%$ \\
0.7 & 0 & 259.7954 & $1.49 \%$ \\
0.8 & 0 & 241.6133 & $1.39 \%$ \\
0.9 & 0 & 247.4119 & $1.42 \%$ \\
1.0 & 0 & &
\end{tabular}

distributed load. We can see that the std for the reserve adjustment approach is almost an order of magnitude higher than that for the adaptive robust approach (8.15-14.48 times). The significant reduction in the std of the dispatch cost is closely related to the significant reduction in the penalty cost. Table IV shows the penalty costs of the two approaches. Recall that the dispatch cost is the sum of the production cost and penalty cost. The penalty cost occurs whenever there is a violation in the energy balance, reserve requirement, or transmission constraints. The system operator has to take expensive emergency actions such as dispatching fast-start units or load-shedding to maintain system reliability. All these add volatility to the dispatch costs. Tables I-III also provide a tool for benefit-risk analysis. In particular, for different levels of uncertainty budget, Tables I and II characterize the economic efficiency obtained by the robust solutions, whereas Table III shows the risk of the robust solutions in terms of the standard deviations of the cost. We can observe that lower uncertainty budget leads to better economic benefit, but worse risk performance. Using these tables, a proper tradeoff can be made by decision makers.

As we observe, all three types of constraint violations (energy, reserve, and transmission) may occur for ResAdj solutions, indicating the potential ineffectiveness of a deterministic approach that only considers nominal load and system level reserves. In contrast, the AdptRob approach commits resources by taking consideration of all possible load realizations in the uncertainty model. Furthermore, the robust solutions remain feasible even when the load realization is outside of the uncertainty set (as the case for normally distributed load).

In conclusion, the low volatility of the dispatch cost and the zero penalty cost of the adaptive robust approach demonstrates

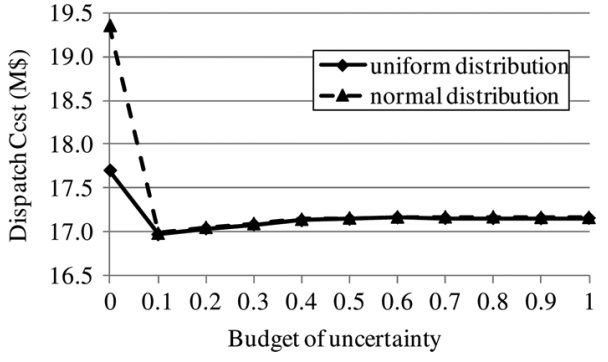

Fig. 2. Average dispatch costs of the AdptRob approach for normally and uniformly distributed load.

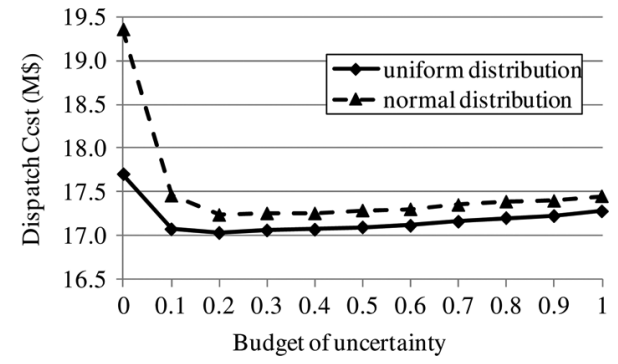

Fig. 3. Average dispatch costs of the ResAdj approach for normally and uniformly distributed load.

its operational effectiveness in reducing costly emergency actions and improving system reliability.

\section{Robustness Against Load Distributions}

In practice, it is not easy to accurately identify the probability distribution of the load uncertainty for each node, especially in a large-scale power system. Thus, it is important for a UC solution to have stable economic and operational performance over different distributions of the uncertain load. The simulation results show that the adaptive robust approach exhibits this desirable property. In comparison, the performance of the reserve adjustment approach is significantly affected by the underlying probability distribution.

As shown in Fig. 2, the average dispatch costs of the AdptRob approach are almost the same for loads with normal and uniform distributions. The absolute difference between the two curves is between $\$ 6.32 \mathrm{k}$ and $\$ 15.80 \mathrm{k}$ for the entire range of $\Delta^{t}=$ $0.1 N_{d}, \ldots, 0.9 N_{d}$. The relative difference is between $0.037 \%$ to $0.092 \%$ [defined as (normal cost-uniform cost)/normal cost].

The ResAdj approach has a rather different picture, as shown in Fig. 3. The average dispatch costs are significantly affected by the load probability distribution. The absolute difference of the two curves varies between $\$ 174.42 \mathrm{k}$ and $\$ 382.26 \mathrm{k}$. The relative difference is between $1.0 \%$ to $2.2 \%$. In both measures, the difference is more than 20 times larger than that of the AdptRob approach.

We also study the effect of the load distribution on the standard deviation of dispatch costs. Table $\mathrm{V}$ shows the std of dispatch costs for loads with uniform distribution and the relative difference between the uniform distribution and the normal distribution (defined as (normal std—uniform std)/normal std, cf. Table III for normal std). As shown in the table, the relative change of the std is around $18.8 \%$ for the AdptRob approach, and is around $59.6 \%$ for the ResAdj approach, which is more 
TABLE V

STANDARD DEVIATION OF DisPaTCH COSTS FOR UNIFORMLY DisTRIBUTED LOAD AND THE RELATIVE DIFFERENCE With THOSE OF THE NORMALLy Distributed LOAD

\begin{tabular}{ccc|cc}
\hline \multicolumn{4}{c|}{ AdptRob } & \multicolumn{2}{c}{ ResAdj } \\
\hline budget & std. disp. & Relat. & std. disp. & relat. \\
$\Delta^{t} / N_{d}$ & cost (k\$) & diff & cost (k\$) & diff \\
\hline 0.0 & 624.5939 & $64.70 \%$ & 624.5939 & $64.70 \%$ \\
0.1 & 38.5824 & $18.76 \%$ & 227.7822 & $66.87 \%$ \\
0.2 & 37.6948 & $18.70 \%$ & 153.5149 & $61.61 \%$ \\
0.3 & 36.8956 & $18.78 \%$ & 157.0428 & $58.43 \%$ \\
0.4 & 36.2507 & $18.06 \%$ & 150.7548 & $58.89 \%$ \\
0.5 & 35.7960 & $18.84 \%$ & 156.9203 & $58.40 \%$ \\
0.6 & 35.6687 & $18.92 \%$ & 154.4555 & $58.35 \%$ \\
0.7 & 35.6179 & $18.91 \%$ & 156.8091 & $58.41 \%$ \\
0.8 & 35.6067 & $18.95 \%$ & 154.3305 & $58.37 \%$ \\
0.9 & 35.5761 & $18.97 \%$ & 148.2292 & $58.59 \%$ \\
1.0 & 35.6092 & $18.97 \%$ & 151.8466 & $57.94 \%$
\end{tabular}

TABLE VI

Average Dispatch Costs and Total Costs of the ADPTROB AND RESADJ FOR NORMALLY DISTRIBUTED LOAD FOR $\Delta^{t} / N_{d}=0,0.1, \ldots, 1$ AND $\hat{d}_{j}^{t}=0.2 \bar{d}_{j}^{t}$

\begin{tabular}{c|cc|cc}
\hline & \multicolumn{2}{|c|}{ AdptRob } & \multicolumn{2}{c}{ ResAdj } \\
\hline $\begin{array}{c}\text { budget } \\
\Delta^{t} / N_{d}\end{array}$ & $\begin{array}{c}\text { dispatch cost } \\
\text { (MS) }\end{array}$ & $\begin{array}{c}\text { total cost } \\
\text { (M\$) }\end{array}$ & $\begin{array}{c}\text { dispatch cost } \\
\text { (M\$) }\end{array}$ & $\begin{array}{c}\text { total cost } \\
\text { (M\$) }\end{array}$ \\
\hline 0.0 & 33.5377 & 35.0589 & 33.5377 & 35.0589 \\
0.1 & 17.4093 & 19.2498 & 19.8504 & 21.3521 \\
0.2 & 17.6524 & 19.5839 & 18.6280 & 20.1553 \\
0.3 & 17.8603 & 19.9179 & 18.5842 & 20.1357 \\
0.4 & 17.9390 & 20.2108 & 18.6651 & 20.2224 \\
0.5 & 18.4717 & 21.2104 & 18.5935 & 20.2164 \\
0.6 & 18.3158 & 20.9412 & 18.4244 & 20.1446 \\
0.7 & 19.2184 & 22.2851 & 18.8700 & 20.8222 \\
0.8 & 19.7684 & 23.1764 & 24.5734 & 26.7734 \\
0.9 & 18.5987 & 21.2892 & 39.3593 & 42.4111 \\
1.0 & 18.5811 & 21.2944 & 57.3552 & 61.6723 \\
\hline
\end{tabular}

than three times higher. It is also interesting to note that the AdptRob approach significantly reduces the relative change of the std comparing to the deterministic approach $\left(\Delta^{t}=0\right)$ from $64.70 \%$ to around $18.8 \%$. On the other hand, the ResAdj approach is much less effective in this respect (from $64.70 \%$ to around $59.6 \%$ ).

\section{Cases for Higher Level of Load Variation}

We also test the performance of the robust adaptive UC model at a higher level of load variation, namely $\hat{d}_{j}^{t}=0.2 \bar{d}_{j}^{t}$. The comparison with the corresponding results of the reserve adjustment approach reveals further advantages of the robust approach in cost saving and volatility reduction, as well as the limitation of the reserve approach in dealing with high level of demand variation.

Table VI shows the average dispatch and total costs of the two approaches. Firstly, we can see that the AdptRob model has relatively stable dispatch and total costs across the entire range of budget levels (around \$17-19 million for dispatch cost and \$19-23 million for total costs), while the ResAdj model incurs significantly higher costs at budget levels $\Delta^{t} \geq 0.8 N_{d}$ (dispatch and total costs rise up to $\$ 57$ million and $\$ 61$ million, respectively). These extremely high costs are mainly due to the fact that there are not enough reserves in the system to be procured to meet the higher reserve requirement associated with the higher budget levels during the unit commitment procedure. In turn, significant balance and transmission violations occur in
TABLE VII

Standard DeViation of the Dispatch Costs of the Two Approaches AND THEIR RATIO FOR NORMALLY DISTRIBUTED LOAD AND $\hat{d}_{j}^{t}=0.2 \bar{d}_{j}^{t}$

\begin{tabular}{c|ccc}
\hline & AdptRob & ResAdj & ResAdj \\
\hline budget & $\begin{array}{c}\text { std dispatch } \\
\text { cost }(\mathrm{k} \$)\end{array}$ & $\begin{array}{c}\text { std dispatch } \\
\text { cost }(\mathrm{k} \$)\end{array}$ & $\begin{array}{c}\text { / } \\
\text { AdptRob }\end{array}$ \\
$\Delta^{t} / N_{d}$ & $6,839.5245$ & $6,839.5245$ & 1.00 \\
0.0 & 132.3089 & $2,407.9004$ & 18.20 \\
0.1 & 187.0653 & $1,374.5625$ & 7.35 \\
0.2 & 132.9050 & $1,275.8496$ & 9.60 \\
0.3 & 130.8215 & $1,267.6123$ & 9.69 \\
0.4 & 132.6666 & $1,193.7796$ & 9.00 \\
0.5 & 131.8476 & $1,020.5538$ & 7.74 \\
0.6 & 135.5654 & $1,732.0398$ & 12.78 \\
0.7 & 138.6719 & $7,276.9223$ & 52.48 \\
0.8 & 131.9013 & $13,480.0202$ & 102.20 \\
0.9 & 132.1308 & $\mathbf{1 8 , 2 4 0 . 1 5 8 5}$ & 138.05 \\
1.0 & & &
\end{tabular}

TABLE VIII

Penalty Costs of AdPTRob AND ResAdj Approaches FOR NORMALLY DISTRIBUTED LOAD AND $\hat{d}_{j}^{t}=0.2 \bar{d}_{j}^{t}$

\begin{tabular}{c|cc|cc}
\hline & \multicolumn{2}{|c}{ AdptRob } & \multicolumn{2}{c}{ ResAdj } \\
\hline budget & $\begin{array}{c}\text { penalty } \\
\Delta^{t} / N_{d}\end{array}$ & $\begin{array}{c}\text { percent of } \\
\text { cost }(\mathrm{k} \$)\end{array}$ & $\begin{array}{c}\text { penalty } \\
\text { disp. cost }\end{array}$ & $\begin{array}{c}\text { percent of }(\mathrm{k} \$) \\
\text { disp. cost }\end{array}$ \\
\hline 0.0 & $16,438.9480$ & $49.02 \%$ & $16,438.9480$ & $49.02 \%$ \\
0.1 & 9.4449 & $0.054 \%$ & $2,775.2887$ & $13.98 \%$ \\
0.2 & 20.7858 & $0.118 \%$ & $1,535.3607$ & $8.24 \%$ \\
0.3 & 10.1540 & $0.057 \%$ & $1,447.1310$ & $7.79 \%$ \\
0.4 & 8.9565 & $0.050 \%$ & $1,442.5843$ & $7.73 \%$ \\
0.5 & 8.9565 & $0.048 \%$ & $1,302.5751$ & $7.01 \%$ \\
0.6 & 8.9565 & $0.049 \%$ & 947.9048 & $5.14 \%$ \\
0.7 & 8.9565 & $0.047 \%$ & $1,158.2852$ & $6.14 \%$ \\
0.8 & 8.9565 & $0.045 \%$ & $6,162.2991$ & $25.07 \%$ \\
0.9 & 8.9565 & $0.048 \%$ & $19,302.3638$ & $49.04 \%$ \\
1.0 & 8.9565 & $0.048 \%$ & $35,412.5130$ & $61.74 \%$ \\
\hline
\end{tabular}

the simulation under such ResAdj UC solutions. This indicates a limit on the demand uncertainty levels that a system operator can safely deal with by relying on the ResAdj approach. Secondly, in the range where the ResAdj approach has reasonable costs, Table VI shows that the AdptRob model has a dispatch cost saving of $12.3 \%$ or $\$ 2.44$ million (at $\Delta^{t}=0.1 N_{d}$ ) and total cost saving of $9.8 \%$ or $\$ 2.10$ million, which is a much higher saving than the previous results $(6.96 \%$ or $\$ 1.27$ million for dispatch costs and $5.48 \%$ or $\$ 1.08$ million for total costs at a lower level of demand deviation, see Section V-A).

Table VII shows the std of dispatch costs of the two approaches. Again, we can see that the ResAdj approach has extremely high std on dispatch costs at high reserve adjustment levels $\Delta^{t} \geq 0.8 N_{d}$ due to high levels of violations. Even in the range of $\Delta^{t}=0.1 \sim 0.7 N_{d}$, the ResAdj approach have from 7.35 times to 18.20 times higher std on dispatch costs than the AdptRob approach. Comparing to the previous results of $8.14 \sim 14.48$ times higher std (see Table III), this demonstrates that the AdptRob approach has an even more significant reduction in cost volatility at higher level of demand variation. Table VIII lists the penalty costs of the two approaches, where the penalty costs of the AdptRob approach are on average less than $0.06 \%$ of the dispatch costs, while the numbers are more than two orders of magnitude higher for the ResAdj approach (from $5.14 \%$ to $13.98 \%$ for $\Delta^{t} \leq 0.7 N_{d}$, and as high as $61.74 \%$ for $\Delta^{t} \geq 0.8 N_{d}$ ).

We also study the robustness of the AdptRob approach at the higher level of demand variation. To be compact, we omit 
the plots and tables, but summarize the key statistics. In particular, the AdptRob approach exhibits similar robustness to underlying distributions: the absolute difference of dispatch costs under normal and uniform distributions is between $\$ 23.70 \mathrm{k}$ and $\$ 98.92 \mathrm{k}$ for the entire range of $\Delta^{t}=0.1 N_{d}, \ldots, 1.0 N_{d}$, or between $0.14 \%$ and $0.55 \%$ in terms of relative difference. The ResAdj approach is much more sensitive to the distribution: the absolute difference is between $\$ 456.91 \mathrm{k}$ and $\$ 8,435.23 \mathrm{k}$, and the relative difference is between $2.48 \%$ and $23.08 \%$. Similarly, the std of dispatch costs of the AdptRob approach is more robust: the absolute difference under two distributions is between $\$ 60.34 \mathrm{k}$ and $\$ 65.01 \mathrm{k}$, or relative difference between $43.5 \%$ and $47.7 \%$ for the AdptRob approach, and the absolute difference is between $\$ 385.5 \mathrm{k}$ and $\$ 4,913.2 \mathrm{k}$, or relative difference between $21.78 \%$ and $47.53 \%$ for the ResAdj approach.

\section{CONCLUSION AND Discussion}

The adaptive robust model and its solution technique presented in this paper provide a novel and practical approach to handle uncertainties in the unit commitment process. Such a framework naturally fits into the daily reliability unit commitment process in an ISO environment. We develop a practical solution method with the real-world large scale power system operation in mind. We conduct extensive tests on the large scale system operated by the ISO New England, and compare our model with the current reserve adjustment approach. We find that by properly setting the level of conservatism in the uncertainty model, the adaptive robust model exhibits sizable savings on both average dispatch and total costs, and significantly reduces the volatility of the dispatch cost, thus, improves the real-time reliability of the power system operation. The robust model also shows resilient performance under different probability distributions of load. The advantages of the adaptive robust model are further illustrated by numerical tests on the same large scale system with higher level of net injection uncertainty ( $20 \%$ deviation range from the nominal net injection levels).

Some discussions are in order. The proposed robust UC model is designed for the reliability unit commitment (RUC) phase in the daily market operation. The RUC phase is carried out by the ISO after the clearance of the day-ahead energy market. The purpose is to evaluate the reliability of the day-ahead market solution against forecast load and contingencies, and to commit additional resources if necessary. RUC is an indispensable step for ensuring reliability and security of the power system. Electricity prices should still be set in the day-ahead market, which functions as a purely financial market.

The proposed robust model includes basic reserve requirement in order to cover generator contingencies, since generator contingency is not considered in the uncertainty model. The computational results show that the robust approach is economically efficient in reducing additional reserve requirement, when the net nodal injection is uncertain.

The stochastic factors that influence the unit commitment problem are associated with both supply (e.g., wind power, etc.) and demand (e.g., demand forecast errors and price responsive demand). To address these various uncertainty elements, the proposed two-stage adaptive robust framework models the uncertainty at the individual nodal level. Therefore, the impact of resource level uncertainty on the transmission system can be evaluated. Moreover, the proposed model can be readily extended to include uncertainties related to inter-tie exchanges, system-wide and zonal level load, and interface limit de-rating.

In our model, we assume that the commitment cost function and the dispatch cost function are both linear, which is a common assumption in the UC literature. In more realistic settings, the production cost can be modeled as a quadratic function, the start-up cost can be described by an exponential function, both of which can be approximated by piecewise linear functions and readily incorporated in our model. A nonconvex cost function can also be approximated by a nonconvex piecewise linear function with binary variable techniques as shown in [27]. The outer-approximation algorithm needs to be modified in this case and the performance of such reformulation is subject to detailed experimentation.

The framework of the proposed solution methodology, especially the outer approximation technique to solve the second-stage problem, is not restricted to the budgeted uncertainty set and can be applied to general polyhedral uncertainty sets. The methodology can also be generalized to handle nonlinear convex constraints, such as ellipsoidal uncertainty sets, which have been used to model correlations between uncertain variables; see [19]. We can also use a set of underlying common factors to model the correlation between uncertain variables. This approach has the advantage of allowing polyhedral uncertainty sets, which reduces computational complexity to solve the large-scale mixed integer robust unit commitment problem. We also remark that the second-stage problem can be alternatively formulated as a mixed-integer optimization problem, using the common technique of linearization of the bilinear term. However, in our experiments, we observe slow convergence of this formulation for large scale problems.

In the current practice, the SFT runs iteratively with the unit commitment procedure by gradually adding violated transmission security constraints to the economic dispatch problem. Alternatively, as we implemented in our numerical test, we can impose a set of critical transmission security constraints in the second-stage problem without running the SFT. These critical transmission security constraints are more likely to be violated than other constraints based on expert knowledge and historical data, and they are usually a small subset of the total transmission constraints. Therefore, this alternative approach can reduce the computation time for solving the second-stage problem. To demonstrate the performance of the proposed algorithm in handling more transmission lines, we conducted further experiments where 1876 transmission constraints are modeled under the normal and contingency conditions. For all levels of uncertainty budget, the algorithm is able to solve the problem to the same level of accuracy as set for the previous experiments. The average computation time is $8.21 \mathrm{~h}$ on the PC laptop for the high accuracy setting (see specification in Section V).

Many interesting directions are open for future research. For example, it would be interesting to study re-commitment that is adaptive to load forecast. We can easily adjust the parameters such as $\hat{d}_{j}^{t}$ in the uncertainty set and re-run our model for future re-commitment when a better estimation of uncertainty is 
available. This could be very useful when the system has high percentage of price responsive demand and variable supply. We already show that the robust solution significantly reduces the volatility of the dispatch cost. It would be interesting to study the extent that the volatility in the energy price is reduced. It would also be very interesting to model explicit correlation between different uncertain loads, such as spatial correlation between adjacent wind farms, or temporal correlation between consumption levels. Such correlation can be captured by modeling covariance matrices in the uncertainty set. Developing other robust models with different decision rules (such as affinely adjustable dispatch decision [26]) is another on-going project. Another interesting research direction is to consider unexpected generator outages in the robust model. The second stage dispatch solution is adaptive to not only the demand uncertainty, but also generator outage uncertainty. How to build the associated uncertainty set and how to deal with the more complicated robust counterpart are important questions in this direction. Furthermore, studying the impact on reserve requirement also has practical significance.

\section{APPENDIX}

For the unit commitment decision making, the system operator usually has access to a wide range of detailed data listed below, including economic data of generator's production costs or supply curve in a market setting, physical characteristics of each generator, expected load forecast, system reserve requirement, network parameters and transmission line ratings.

- $N_{g}, N_{d}, N_{b}, N_{l}, T$ : The number of generators, loads, nodes, transmission lines, and time periods (in hours).

- $\mathcal{N}_{g}, \mathcal{N}_{d}, \mathcal{N}_{b}, \mathcal{N}_{l}, \mathcal{T}, \mathcal{C} \mathcal{T}_{i}$ : The corresponding sets of generators, loads, nodes, transmission lines, time periods (in hours), and available transmission lines when line $i$ is tripped.

- $S_{i}^{t}, G_{i}^{t}, F_{i}^{t}$ : Start-up, shut-down, and no-load costs of generator $i$ at time $t$.

- $C_{i}^{t}(\cdot)$ : Variable cost of generator $i$ at time $t$ as a function of production levels.

- $p_{i}^{\max }, p_{i}^{\min }$ : Maximum and minimum production levels of generator $i$ (usually called Ecomax and Ecomin, respectively).

- $R U_{i}^{t}, R D_{i}^{t}$ : Ramp-up and ramp-down rates of generator $i$ at time $t$.

- $\operatorname{MinUp}_{i}, \mathrm{MinDw}_{i}$ : Minimum-up and minimum-down times of generator $i$.

- $f_{l}^{\max }$ : Flow limit on transmission line $l$ in base case.

- $f_{l, i}^{\max }$ : Flow limit on transmission line $l$ in contingency $i$ (i.e., line $i$ is dropped).

- $\boldsymbol{B}_{p}, \boldsymbol{B}_{d}$ : Network incidence matrices for generators and load.

- $\boldsymbol{a}_{l}$ : Network shift factor vector for line $l$ for the base case.

- $\boldsymbol{a}_{l, i}$ : Network shift factor vector for line $l$ in contingency $i$.

- $\bar{d}_{j}^{t}$ : Expected demand at node $j$, time $t$.

- $\mathcal{N}_{r}$ : The set of system reserve requirements $\mathcal{N}_{r}=$ \{TMSR, T10, T30\}.

- $\mathcal{A}$ : The set of reserve products $\mathcal{A}=$ \{TMSR, TMNSR, TMOR\}.
- $\mathcal{A}_{k}$ : The set of reserve products needed to satisfy reserve requirement $k \in \mathcal{N}_{r}: \mathcal{A}_{\mathrm{TMSR}}=$ $\{\mathrm{TMSR}\}, \mathcal{A}_{\mathrm{T} 10}=\{\mathrm{TMSR}, \mathrm{TMNSR}\}, \mathcal{A}_{\mathrm{T} 30}=$ \{TMSR, TMNSR, TMOR $\}$.

- $\bar{q}_{i, k}^{t}:$ Reserve capacity of generator $i$, requirement $k \in \mathcal{N}_{r}$, time $t$.

- $\bar{q}_{k}^{t}$ : System reserve requirement of $k \in \mathcal{N}_{r}$, time $t$.

The system operator in the current practice commits extra generation resource to provide reserves in the day-ahead scheduling. The reserve capacity will be available to the system operator in the real-time operation to prepare for unexpected loss of generators or other system disruptions. According to how fast the reserve capacity can respond to the emergency, there are three important types of reserves: ten-minute spinning reserve (TMSR), ten-minute nonspinning reserve (TMNSR), and thirty-minute operating reserve (TMOR). Other types of reserves exist, such as regulation service (automatic generation control) which responds to frequency changes in the system second by second, and supplement reserve.

The decision variables of the unit commitment problem are as follows.

- $x_{i}^{t} \in\{0,1\}$ : If generator $i$ is on at time $t, x_{i}^{t}=1$; otherwise $x_{i}^{t}=0$.

- $u_{i}^{t} \in\{0,1\}$ : If generator $i$ is turned on at time $t, u_{i}^{t}=1$; otherwise $u_{i}^{t}=0$.

- $v_{i}^{t} \in\{0,1\}$ : If generator $i$ is turned down at time $t, v_{i}^{t}=1$; otherwise $v_{i}^{t}=0$.

- $p_{i}^{t} \in[0, \infty)$ : Production of generator $i$ at time $t$.

- $q_{i, a}^{t} \in[0, \infty)$ : Reserve of generator $i$, type $a \in \mathcal{A}$, time $t$.

A standard deterministic UC model is formulated below [24], [25]:

$$
\begin{aligned}
& \min _{\boldsymbol{x}, \boldsymbol{u}, \boldsymbol{v}, \boldsymbol{p}, \boldsymbol{q}} \sum_{t=1}^{T} \sum_{i=1}^{N_{g}} x_{i}^{t} F_{i}^{t}+u_{i}^{t} S_{i}^{t}+v_{i}^{t} G_{i}^{t}+C_{i}^{t}\left(p_{i}^{t}\right) \\
& \text { s.t. } \quad x_{i}^{t-1}-x_{i}^{t}+u_{i}^{t} \geq 0, \quad \forall i \in \mathcal{N}_{g}, t \in \mathcal{T} \\
& x_{i}^{t}-x_{i}^{t-1}+v_{i}^{t} \geq 0, \quad \forall i \in \mathcal{N}_{g}, t \in \mathcal{T} \\
& x_{i}^{t}-x_{i}^{t-1} \leq x_{i}^{\tau} \\
& \forall \tau \in\left[t+1, \min \left\{t+\operatorname{MinUp}_{i}-1, T\right\}\right], t \in[2, T], \\
& x_{i}^{t-1}-x_{i}^{t} \leq 1-x_{i}^{\tau} \\
& \forall \tau \in\left[t+1, \min \left\{t+\operatorname{MinDw}_{i}-1, T\right\}\right], t \in[2, T], \\
& \sum_{i=1}^{N_{g}} p_{i}^{t}=\sum_{j=1}^{N_{d}} \bar{d}_{j}^{t}, \quad \forall t \in \mathcal{T} \\
& -R D_{i}^{t} \leq p_{i}^{t}-p_{i}^{t-1} \leq R U_{i}^{t}, \quad \forall i \in \mathcal{N}_{g}, t \in \mathcal{T} \\
& -f_{l}^{\max } \leq \boldsymbol{a}_{l}^{\prime}\left(\boldsymbol{B}_{p} \boldsymbol{p}^{t}-\boldsymbol{B}_{d} \boldsymbol{d}^{t}\right) \leq f_{l}^{\max } \\
& \forall t \in \mathcal{T}, l \in \mathcal{N}_{l}, \\
& -f_{l, i}^{\max } \leq \boldsymbol{a}_{l, i}^{\prime}\left(\boldsymbol{B}_{p} \boldsymbol{p}^{t}-\boldsymbol{B}_{d} \boldsymbol{d}^{t}\right) \leq f_{l, i}^{\max } \\
& \forall t \in \mathcal{T}, l \in \mathcal{C T}_{i}, i \in \mathcal{N}_{l}, \\
& p_{i}^{\min } \leq p_{i}^{t}+\sum_{a \in \mathcal{A}} q_{i, a}^{t} \leq p_{i}^{\max }, \quad \forall i \in \mathcal{N}_{g}, t \in \mathcal{T} \\
& p_{i}^{\min } x_{i}^{t} \leq p_{i}^{t} \leq p_{i}^{\max } x_{i}^{t}, \quad \forall i \in \mathcal{N}_{g}, t \in \mathcal{T} \\
& \sum_{i \in \mathcal{N}_{g}, a \in \mathcal{A}_{k}} q_{i, a}^{t} \geq \bar{q}_{k}^{t}, \quad \forall t \in \mathcal{T}, k \in \mathcal{N}_{r} \\
& \sum_{a \in \mathcal{A}_{k}} q_{i, a}^{t} \leq \bar{q}_{i, k}^{t}, \quad \forall i \in \mathcal{N}_{g}, t \in \mathcal{T}, k \in \mathcal{N}_{r} .
\end{aligned}
$$


Equations (13) and (14) are logic constraints between on and off status and the turn-on and turn-off actions. In particular, a generator $i$ is turned on at time $t u_{i}^{t}=1$ if and only if $x_{i}^{t-1}=$ $0, x_{i}^{t}=1$. Similarly, a generator $i$ is turned off at time $t$ if and only if $x_{i}^{t-1}=1, x_{i}^{t}=0$. Equations (15) and (16) are constraints of minimum up and minimum down times for each generator, i.e., if a generator is turned on at time $t$, then it must remain on at least for the next $\left(\mathrm{MinUp}_{i}-1\right)$ periods, and similar for the shutdown constraint. There are multiple ways to model these constraints. The specific form that we use here follow the formulation proposed in [28].

Equation (17) is the energy balance equation that matches the system level supply and load at each time period. Equation (18) is the ramp rate constraint, i.e., the speed at which a generator can increase or decrease its production level is bounded in a range. Notice that the ramping constraint is a complicating constraint that couples many consecutive time periods. Also notice that the ramp constraints should consider the case that a generator's output level should not be limited by the ramp rate, when it is started at the beginning of a period. Such complicating situations are considered in our code implementation. But (18) captures the essence of ramp constraints and thus is presented here for simplicity purpose.

Equation (19) is the transmission flow constraint for the base case, where all transmission lines are functioning. Equation (20) is the transmission line constraint for the $i$ th contingency where transmission line $i$ is tripped. In this situation, the network topology is changed, so are the shift factor $\boldsymbol{a}_{l, i}$ and flow limits $f_{l, i}^{\max }$.

Equation (21) is the constraint that the sum of the production output and the reserve should be within the upper and lower bounds for each generator. Equation (22) is the coupled constraint indicating that only committed generators can be dispatched. Equation (23) describes the requirement on how much reserve the system should have for reserve category $k \in \mathcal{N}_{r}$ at time $t$. Equation (24) says generator $i$ can provide at most $\bar{q}_{i, k}^{t}$ for reserve requirement $k$ at time $t$.

The variable production cost, or the supply curve in a market setting, $C_{i}^{t}\left(p_{i}^{t}\right)$ is an increasing convex piece-wise linear function of the production output $p_{i}^{t}$.

\section{REFERENCES}

[1] L. T. Anstine, R. Burke, J. E. Casey, R. Holgate, R. John, and H. G. Stewart, "Application of probability methods to the determination of spinning reserve requirements for Pennsylvania-New Jersey-Maryland," IEEE Trans. Power App. Syst., vol. PAS-82, no. 68, pp. 726-735, 1963.

[2] R. Billinton and R. Karki, "Capacity reserve assessment using system well-being analysis," IEEE Trans. Power Syst., vol. 14, pp. 433-438, 1999.

[3] R. Billinton and M. Fotuhi-Firuzabad, "A reliability framework for generating unit commitment," Elect. Power Syst. Res., vol. 56, no. 1, pp. $81-88,2000$.

[4] H. Gooi, D. Mendes, K. Bell, and D. Kirschen, "Optimum scheduling of spinning reserve," IEEE Trans. Power Syst., vol. 14, pp. 1485-1490, 1999.

[5] P. Carpentier, G. Gohen, J. C. Culioli, and A. Renaud, "Stochastic optimization of unit commitment: A new decomposition framework," IEEE Trans. Power Syst., vol. 11, pp. 1067-1073, 1996.

[6] S. Takriti, J. R. Birge, and E. Long, "A stochastic model for the unit commitment problem," IEEE Trans. Power Syst., vol. 11, pp. $1497-1508,1996$
[7] U. Ozturk, M. Mazumdar, and B. Norman, "A solution to the stochastic unit commitment problem using chanced constrained programming," IEEE Trans. Power Syst., vol. 19, no. 3, pp. 1589-1598, Aug. 2005.

[8] L. Wu, M. Shahidehpour, and T. Li, "Stochastic security-constrained unit commitment," IEEE Trans. Power Syst., vol. 22, pp. 800-811, 2007.

[9] S. Wong and J. Fuller, "Pricing energy and reserves using stochastic optimization in an alternative electricity market," IEEE Trans. Power Syst., vol. 22, no. 2, pp. 631-638, May 2007.

[10] P. Ruiz, C. Philbrick, E. Zak, K. Cheung, and P. Sauer, "Uncertainty management in the unit commitment problem," IEEE Trans. Power Syst., vol. 24, no. 2, pp. 642-651, May 2009.

[11] E. M. Constantinescu, V. M. Zavala, M. Rocklin, S. Lee, and M. Anitescu, "A computational framework for uncertainty quantification and stochastic optimization in unit commitment with wind power generation," IEEE Trans. Power Syst., vol. 26, no. 1, pp. 431-441, Feb. 2010

[12] A. Ben-Tal and A. Nemirovski, "Robust convex optimization," Math. Oper. Res., vol. 23, pp. 769-805, 1998

[13] A. Ben-Tal and A. Nemirovski, "Robust solutions of uncertain linear programs," Oper. Res. Lett., vol. 25, no. 1, pp. 1-13, 1999.

[14] A. Ben-Tal and A. Nemirovski, "Robust solutions of linear programming problems contaminated with uncertain data," Math. Program. vol. 88 , pp. 411-421, 2000.

[15] L. E. Ghaoui and H. Lebret, "Robust solutions to least-squares problems with uncertain data," SIAM J. Matrix Anal. Appl., vol. 18, pp. $1035-1064,1997$.

[16] L. E. Ghaoui, F. Oustry, and H. Lebret, "Robust solutions to uncertain semidefinite programs," SIAM J. Optimiz., vol. 9, no. 1, pp. 33-52, 1998.

[17] D. Bertsimas and M. Sim, "Robust discrete optimization and network flows," Math. Program., vol. 98, pp. 48-71, 2003.

[18] D. Bertsimas and M. Sim, "The price of robustness," Oper. Res., vol. 52, pp. 35-53, 2004.

[19] A. Ben-Tal, L. E. Ghaoui, and A. Nemirovski, Robust Optimization, ser. Princeton Series in Applied Mathematics. Princeton, NJ: Princeton Univ. Press, 2009.

[20] G. L.R. Jiang, M. Zhang, and Y. Guan, Two-Stage Robust Power Grid Optimization Problem 2010, Tech. Rep.

[21] L. Zhao and B. Zeng, Robust Unit Commitment Problem With Demand Response and Wind Energy, University of South Florida, 2010, Tech. Rep.

[22] M. A. Duran and I. E. Grossmann, "An outer-approximation algorithm for a class of mixed-integer nonlinear programs," Math. Program., vol. 36, no. 3, pp. 306-339, 1986

[23] R. Fletcher and S. Leyffer, "Solving mixed integer programs by outer approximation," Math. Program., vol. 66, no. 3, pp. 327-349, 1994

[24] X. Guan, P. Luh, H. Yan, and J. Amalfi, "An optimization based method for unit commitment," Int. J. Elect. Power Energy Syst., vol. 14, no. 6 , pp. 9-17, 1992.

[25] S. Wang, S. Shahidehpour, D. Kirschen, S. Mokhtari, and G. Irisarri, "Short-term generation scheduling with transmission constraints using augmented Lagrangian relaxation," IEEE Trans. Power Syst., vol. 10, pp. 1294-1301, 1995.

[26] E. G. A. Ben-Tal, A. Goryashko, and A. Nemirovski, "Adjustable robust solutions of uncertain linear programs," Math. Program.: Ser. A and $B$, vol. 99 , no. 2, pp. 351-376, 2004.

[27] D. Bertsimas and J. Tsitsiklis, Introduction to Linear Optimization. Nashua, NH: Athena Scientific, 1997.

[28] S. Takriti, B. Krasenbrink, and L. S.-Y. Wu, "Incorporating fuel constraints and electricity spot prices into the stochastic unit commitment problem," Oper. Res., vol. 48, no. 2, pp. 268-280, 2000.

Dimitris Bertsimas (M'11) received the B.S. degree in electrical engineering and computer science at the National Technical University of Athens, Athens, Greece, in 1985, the M.S. degree in operations research and the Ph.D. in applied mathematics and operations research at the Massachusetts Institute of Technology (MIT), Cambridge, in 1987 and 1988, respectively.

$\mathrm{He}$ is currently the Boeing Professor of Operations Research and the codirector of the Operations Research Center at MIT. Since 1988, he has been in the MIT faculty. His research interests include optimization, stochastic systems, data mining, and their application. In recent years, he has worked in robust and adaptive optimization, health care, and finance. He has co-authored more than 120 scientific papers and 3 graduate level textbooks.

Prof. Bertsimas is a member of the National Academy of Engineering, and area editor for optimization in Management Science. He has received numerous research awards including the Farkas prize (2008), the Erlang prize (1996), the SIAM prize in optimization (1996), the Bodossaki prize (1998), and the Presidential Young Investigator award (1991-1996). 
Eugene Litvinov (SM'06) received the B.S. and M.S. degrees from the Technical University, Kiev, Ukraine, and the Ph.D. degree from Urals Polytechnic Institute, Sverdlovsk, Russia.

Currently, he is a Senior Director of Business Architecture and Technology at the ISO New England, Holyoke, MA. His main interests include power system market-clearing models, system security, computer applications to power systems, and information technology.

Xu Andy Sun (M'11) received the B.E. degree in electronic engineering from Tsinghua University, Beijing, China, in 2003 and the M.S. degree in media arts and sciences from Media Laboratory, and the Ph.D. degree in operations research at Massachusetts Institute of Technology (MIT), Cambridge, in 2005 and 2011, respectively.

He spent a year as a post-doctoral researcher at IBM Thomas J. Watson Research Center, Yorktown Heights, NY, before joining, as an Assistant Professor, the H. Milton Stewart School of Industrial and Systems Engineering, Georgia Institute of Technology, Atlanta, in August 2012. His main research interests are robust and adaptive optimization theory and applications in power systems and electricity market design.
Jinye Zhao (M'11) received the B.S. degree from East China Normal University, Shanghai, China, in 2002 and the M.S. degree in mathematics from National University of Singapore in 2004. She received the M.E. degree in operations research and statistics and the Ph.D. degree in mathematics from Rensselaer Polytechnic Institute, Troy, NY, in 2007.

She is a senior analyst at ISO New England, Holyoke, MA. Her main interests are game theory, mathematical programming, and electricity market modeling.

Tongxin Zheng (SM'08) received the B.S. degree in electrical engineering from North China University of Electric Power, Baoding, China, in 1993, the M.S. degree in electrical engineering from Tsinghua University, Beijing, China, in 1996, and the Ph.D. degree in electrical engineering from Clemson University, Clemson, SC, in 1999.

Currently, he is a technical manager at the ISO New England, Holyoke, MA. His main interests are power system optimization and electricity market design. 